
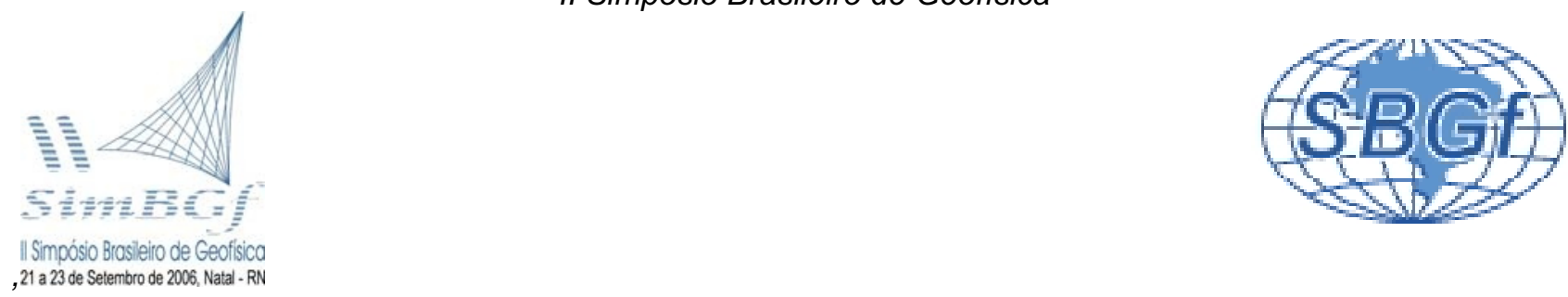

\title{
Structure discrimination using multi-component ground penetrating radar
}

J. M. Travassos, J. E. Musa, Observatório Nacional, Rio de Janeiro, RJ, Brazil

Email: jandyr@on.br, musa@on.br

Copyright 2006, SBGf-Sociedade Brasileira de Geofísica

Este texto foi preparado para a apresentação no II Simpósio de Geofísica da Sociedade Brasileira de Geofísica, Natal, 21-23 de setembro de 2006. Seu conteúdo foi revisado pola Comissão Toco, Nientífica do II SR SBGf mas não necessariamente representa a opinião da SBGf ou de seus associados. E proibida a reprodução total ou parcial deste material para propósitos comerciais sem prévia autorização da SBGf.

\section{Summary}

This work presents some results of a multi-component GPR data set survey done on an area of recent sedimentation, characterized by unconsolidated deposits of eolian, alluvial, lacustrine and marine sands. The data set comprises of a single GPR profile done with two cross-dipole and two copolar antennas configurations. A depolarizing structure situated midrange in TWT in the sections changes the polarization of the incident field giving rise to conspicuous non-zero cross-dipole readings of the scattered field, which displays a linear polarization characteristic. Alford rotation of the data corrected antennas positions in relation to the preferred direction of polarization of the structure thus maximizing the information in the two co-polar components. The rotated data displays a great deal of improvement in imaging capability of the sedimentary environment. A high degree of independence of the two co-polar antennas configurations is also a key factor contributing to that improvement.

Keywords - GPR imaging; multi-component; multipolarization; depolarization effects.

\section{Introduction}

Almost all GPR surveys are performed with a parallelbroadside configuration, also known as the bistatic co-polar configuration, with antennas parallel and perpendicular to the profile. However, it has been known for a while that different configurations can be successfully used for some applications (Annan, 2000). Out of eight possibilities for a bistatic GPR we concentrate on the four antennas configurations shown in Figure 1. The nomenclature used in Figure 1 will be adhered to hereinafter.

The broadside configurations $\left(T_{1} R_{1}\right.$ and $\left.T_{2} R_{2}\right)$ take advantage of the nearly linearly polarized electromagnetic waves, which is parallel to the long axis of the antenna, and thus have the best $S / N$ ratio. On the other hand the crossed-dipole antenna configuration $\left(T_{1} R_{2}\right.$ and $\left.T_{2} R_{1}\right)$ will have the worst $S / N$ ratio as the polarization of the transmitting ( $T)$ and receiving (R) antennas are perpendicular to each other. As a matter of fact we should record a null field with such a configuration on a uniform earth.
Many structures show high degrees of directionality, which are able to change field polarization. They can be either natural such as dipping beds or faults, or man-made such as a buried metallic pipe. A dipping plane will give a different response whether the antennas are oriented parallel or perpendicular in relation to the dip plane. Different antenna configurations may come into play as a focusing mechanism as in the case of depolarizing dipping bodies lying beneath obscuring flat-lying reflectors (Guy and Radzevicius, 1999). In this case a cross-dipole configuration may be the only configuration able to focus on structures below the flat-lying reflectors. Other application is to use a different antenna configuration to reduce the effect of a layer of many diffractors that clutter later times (Travassos and Simões, 2004). As a rule, an antenna configuration different from $\mathrm{T}_{2} \mathrm{R}_{2}$ should be used when the GPR cannot record reflections from subsurface targets either due to a change the polarization of the incident wave or due to clutter at earlier times.

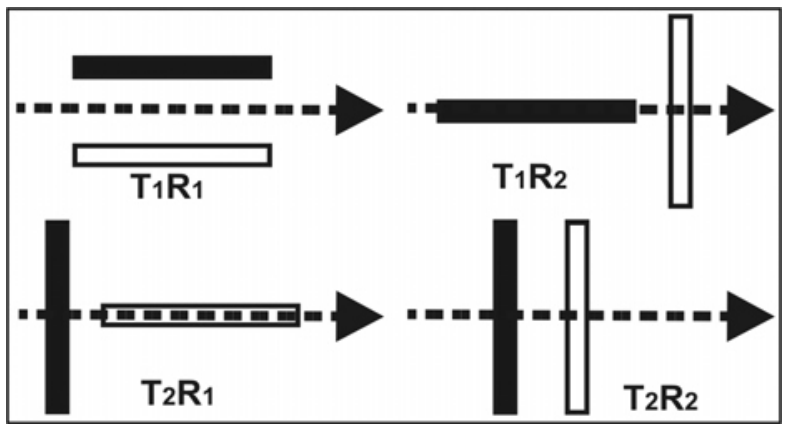

Figure 1. Antenna configurations used in this work: broadside $\left(T_{1} R_{1}\right.$ and $\left.T_{2} R_{2}\right)$, and cross- polarization $\left(T_{1} R_{2}\right.$ and $\left.T_{2} R_{1}\right)$. $T_{i}$ is the transmitting and $R_{j}$ is the receiving antennas, the subscript indicating direction. Profile direction (1) is shown as a dash arrow.

\section{The Experiment}

The experiment was carried out in a University farmland in Santa Catarina Island, southeastern Brazil. The local geology is characterized by unconsolidated deposits of eolian, alluvial, lacustrine and marine sands. The soil is a gray-brown loamy sand on the top of fine grained sand with less than $5 \%$ of silt and clay. Depth to the seasonal water table was $2 \mathrm{~m}$. The horizontal gradient at the site is $1.8 \%$, with a hydraulic conductivity of $10-4 \mathrm{~cm} / \mathrm{s}$ and effective porosity of 0.20 , giving a groundwater flow of $2.8 \mathrm{~m} / \mathrm{yr}$ (Corseuil et al, 2000). 
This work concentrates on a single N-S profile $8 \mathrm{~m}$ long repeated four times, one for each polarization shown in Figure 1. Data was collected with a Pulse Ekko 100 with $200 \mathrm{MHz}$ antennas, 400 ps sampling rate, 32 stack, and 0.1 $\mathrm{m}$ spatial sampling with stop-and-go firing. Topographic control was done with total station.

Data processing was kept to a minimum, comprising of data editing, dewow, an user-defined gaining stage, mild enough not to saturate any trace anywhere, followed by a bandpass filter.

\section{Results}

The electromagnetic field at a given point in space, at the given time, has both a magnitude and a direction, and thus is described by vectors. Polarization describes the magnitude and direction of the electromagnetic (EM) field as a function of time and space. When the time varying EM fields vary sinusoidally (time harmonic), polarization may be classified as linear, circular, or elliptical. The first two are special cases of elliptical polarization, in which the electric field traces out an ellipse.

Three orthogonal basis vectors can describe an arbitrary electromagnetic field. So let the scattered field be described by three orthogonal basis vectors, $\left(\hat{a}_{1}, \hat{a}_{2}, \hat{a}_{3}\right)$, one of which aligned with the direction of wave propagation, say $\hat{a}_{3}$. In this way the propagating electric field is decomposed into the two remaining orthogonal basis vectors $\left(\hat{a}_{1}, \hat{a}_{2}\right)$. Let us assume further a common-offset field configuration with closely spaced antennas so the field at the soil-air interface is back scattered. This exactly the way most GPR surveys are conducted to image the subsurface. In this way the measured scattered electric field is entirely horizontal, and given by

$$
{ }^{R} \vec{E}=\left(\vec{E} \cdot{ }^{R} \hat{a}\right){ }^{R} \hat{a}
$$

where $R_{\hat{a}}$ is a unitary vector and the superscript $\left({ }^{R}\right)$ indicates along the direction of the receiving antenna.

The far-field scattered electric field in (1) can be expressed as a vector sum of two orthogonal components in a Cartesian reference frame $\{1,2\}$ defined by two mutually perpendicular antenna positions and profile direction. This is achieved first measuring the scattered field with transmitter and receiver antennas parallel, T1R1, and then rotating the receiver $90^{\circ}, \mathrm{T} 1 \mathrm{R} 2$,

$$
\vec{E}^{k}={ }^{R} E_{1}^{k R} \hat{a}_{1}+{ }^{R} E_{2}^{k R} \hat{a}_{2}
$$

where $\mathbf{E}^{k}{ }_{i}$ indicates that field amplitude is measured with transmitter along direction $\mathrm{k}\left(\mathrm{T}_{\mathrm{k}}\right)$ and receiver along direction i $\left(\mathrm{R}_{\mathrm{i}}\right)$.

Figure 2 shows the results from the four fixed-offset antennas configurations shown in Figure 1. They were AGC gained just for visualization as signal energy levels are good. The reflections seen in the figure are mainly produced by textural variation within a sequence of sand grain sizes.

We can associate the scalar values of the measured electric field intensity obtained with the four antennas configurations in a second rank tensor $\mathbf{F}$. In this fashion each corresponding data point of each field polarization will be an element of the tensor, in the same order as shown in
Figures 1 and 2, i.e., the main diagonal will be $\left(T_{1} R_{1}, T_{2} R_{2}\right)$, or $\left(E_{1}^{1}, E_{2}^{2}\right)$.

The elements of the secondary diagonal should be equal by the principle of reciprocity, but due to the change of polarity of the scattered field they are not, as seen in Figure 2. With our data set (134 traces and 532 data points) we produced 71288 tensors.

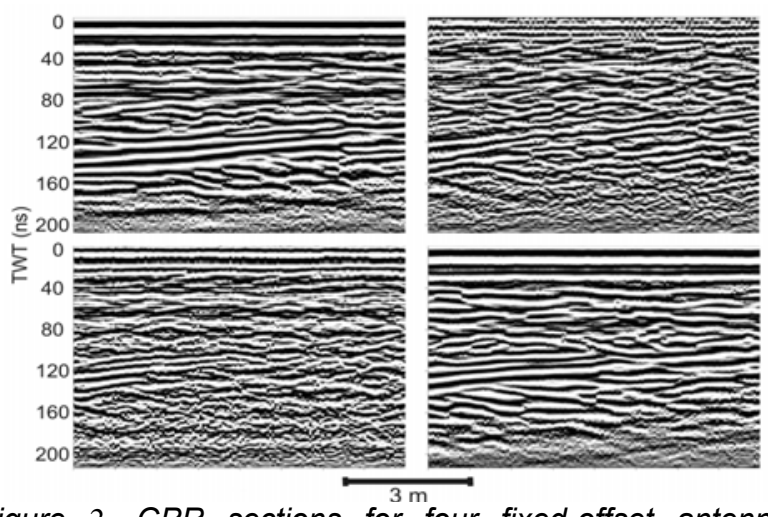

Figure 2. GPR sections for four fixed-offset antennas configurations shown in Figure 1. Sections are displayed in the same order of antenna configuration as in Figure 1.

Each of the tensors can be rotated in relation to the field coordinate system, related to the positions of the antennas and profile direction. This Alford rotation (Van Gestel \& Stoffa, 2001) can produce images equivalent to the copolar or cross-dipole configurations at any given angle of orientation.

Assume a structure able to change the polarization of the incident field towards a preferred direction expressed by the unit vector ${ }^{\mathrm{P}} \hat{\mathrm{a}}$. The incident wave polarization will be vectorially resolved along the direction ${ }^{\mathrm{P}}$ a with a change in polarization of the scattered field, resulting in a non-zero cross-dipole reading. Rotating the tensors to the desired orientation will give the instantaneous amplitude as if the antennas were deployed in the field along, or perpendicular to direction ${ }^{\mathrm{a}} \hat{\mathrm{a}}$. Conversely one can estimate the depolarizing direction ${ }^{P}$ a from the scattered field from the data estimating the angle $\theta_{\mathrm{m}}$ that minimizes the crossdipole readings, thus maximizing the information in the two co-polar configurations.

In our data set most of the estimates of the angle $\theta_{\mathrm{m}}$ are $\leq$ $90^{\circ}$, with peaks at $0^{\circ}, 30^{\circ}-50^{\circ}$, and $80^{\circ}$, Figure 3 . It is possible to realize that the occurrences of $\theta_{\mathrm{m}}$ in the interval $30^{\circ}-50^{\circ}$ fall preferentially in three time zones: $0-20,50-100$, and $>140 \mathrm{~ns}$, Figure 3. Concentrate on TWT = [50-100 ns] interval where there is a clear band of $\theta_{\mathrm{m}}=\left[30^{\circ}-50^{\circ}\right]$ indicating a depolarizing structure with a preferred angle around $40^{\circ}$. The other time zones are of lesser importance as they lie on regions affected by the direct waves, or low signal to noise ratios.

The polarization of the scattered field in the TWT $=[50-$ 100 ns] interval, in rectangle $B$ in Figure 3 , can be assessed analyzing the time-phase difference between the two linearly independent co-polar components, $E_{1}^{1}$ and $E_{2}^{2}$. Figure 4 shows that the vector that describes the harmonically varying electric field varies along a straight 
line, indicating a linear polarization case. Figure 4 was done plotting the two co-polar components of a randomly chosen trace location against each other. The linear polarization is a special case of the more general elliptical polarization.

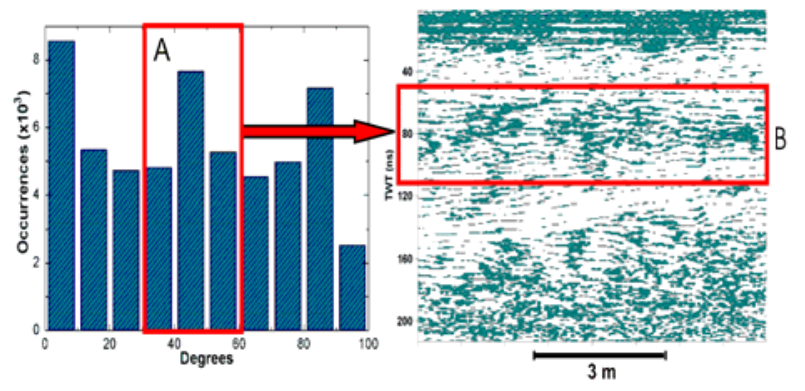

Figure 3. Histogram of the estimates of the angle $\theta_{m} \leq 90^{\circ}$ for our data set. The red rectangle A shows where $\theta_{m}=\left[30^{\circ}\right.$ $-50^{\circ}$. The GPR-like section on the right shows the location of $\theta_{m}$ values that fall inside rectangle $A$. The red rectangle $B$ delineates a structure located in the TWT $=$ [ $50-100 \mathrm{~ns}]$ interval that depolarizes the incident field in a preferred angle around $40^{\circ}$.

Now we are ready to rotate the tensors $\mathbf{F}$ build with our data set, shown in Figure 2. The data inside the rectangle $B$ of Figure 3 is then rotated to $\theta_{\mathrm{m}}=45^{\circ}$. The result is in Figure 5. The improvement in both polarizations is easy to see, with sharper reflectors and complementary information from the two co-polar components. It is also clear that there is a mixing of effects in the sections as there are other influences apart from the depolarizing structure revealed in the angle section of Figure 3.

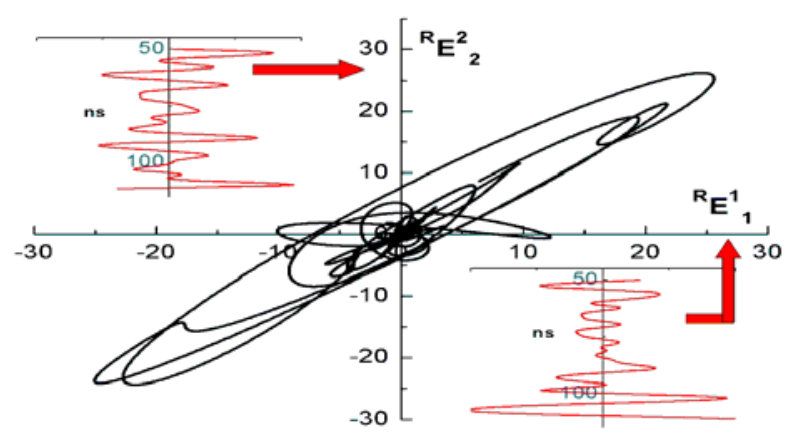

Figure 4: Polarization of the scattered field for a given trace and the $T W T=[50-100 \mathrm{~ns}$ ] interval, shown in the rectangle $B$ of Figure 3. The two co-polar components are plotted against each other. The relevant sections of the traces are shown in the fourth $\left(E_{2}^{2}\right)$ and second $\left(E_{1}^{1}\right)$ quadrants.

The combined effect of the several structures that affect field polarization can be also assessed analyzing the degree of independence between the two co-polar components, $E_{1}^{1}$ and $E^{2}$. That can be done by estimating the two eigenvalues of the tensor $F$, say $\lambda_{1}$ and $\lambda_{2}$. The eigenvalues are rotationally invariant and thus are reference frame invariant. Define a condition number, $k$, as the the ratio of the maximum to the minimum eigenvalue. There are two particular cases: $k=1$ and $k>1$. In the former the two eigenvectors of $\mathbf{F}$ are independent, in the latter they are redundant. Any other value in between will indicate a certain degree of dependence between the two eigenvectors of $\mathbf{F}$.

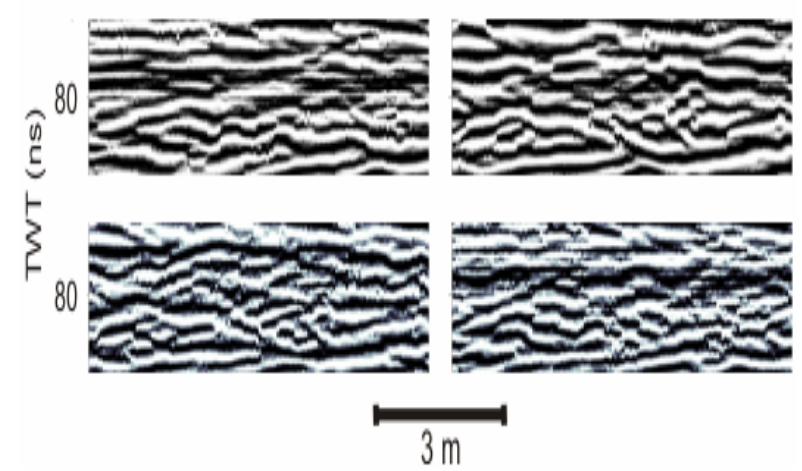

Figure 5. Panel (A) shows an interpretation of co-planar, $T_{1} R_{1}$, and $T_{2} R_{2}$, data for the interval $T W T=[50,100] n s$, shown in the Figure 2. Panel $(B)$ shows the same reflectors seen in $(A)$, but on a $45^{\circ}$ rotated section.

Figure 6 shows that there is a great deal of independence in our data in the TWT $=[50-100$ ns] interval. This indicates that the two co-polar sections are decoupled, i.e., most occurrences are $k \approx 1$. The attention of the reader to the fact that if there were no depolarization effects the two co-polar sections would be decoupled and therefore $k=1$. When depolarization effects are present there is some degree of coupling and $k>1$. As we have shown above we have depolarization effects in the interval indicating there are other intervening structures in the subsurface that do not depolarize the incident field.

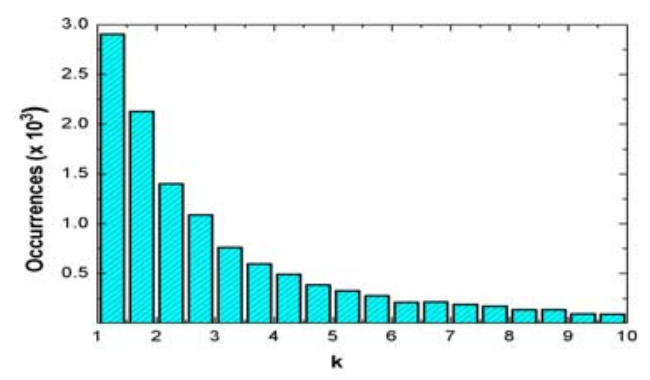

Figure 6: Condition number, $k$, in the TWT $=[50-100$ ns] interval. That interval is shown in the rectangle $B$ of Figure 3.

\section{Conclusion}

We have shown here results obtained with a multipolarization data set obtained on an area of Quaternary sedimentation characterized by unconsolidated deposits of eolian, alluvial, lacustrine and marine sands. The data set comprises of a GPR profile done with two cross-dipole and two co-polar antennas configurations. We have investigated here the angle $\theta_{\mathrm{m}}$ that minimizes the cross-dipole readings and maximizes the information in the two co-polar configurations. 
The angle $\theta_{\mathrm{m}}$ is related to the preferential direction of depolarization of the incident field expressed by a unit vector ${ }^{\mathrm{P}} \mathrm{a}$, caused by a given structure in the subsurface. The incident wave polarization is vectorially resolved along the direction $\mathrm{P}$ â resulting in a change in polarization of the scattered field, giving rise to non-zero cross-dipole readings. As there are more than one intervening structure in the subsurface depolarizing the incident field, the two cross-dipole readings are not equal to each other.

Here we have isolated one depolarizing sub-horizontal structure bounded in the interval TWT $=$ [ $50-100$ ns], which changes the incident field polarization $45^{\circ}$ in relation to the profile direction. The scattered field displays a conspicuous linear polarization. We then applied Alford rotation to the data, maximizing the information in the two co-polar components. Those display a high degree of independence from each other.

With the multi-component GPR processing technique we achieved a great deal of improvement in imaging capability of a sedimentary environment. That was achieved even in our case where data was collected with a field geometry less than optimal, i.e., with a profile direction in an angle in relation of the isolated structure in the interval TWT $=[50-100 \mathrm{~ns}]$. Even considering that improvement it remains the task of combining the multi-component data in a practical way to produce reliable images of the subsurface.

\section{Acknowledgement}

This work was funded by Petrobras. JT is a recipient of a research scholarship from CNPq.

\section{References}

Annan, A.P., Workshop Notes, Sensors \& Software Inc. (2000).

Corseuil, H.X., Fernandes, M., Rosário, M., Seabra, P.N., 2000, Results of a Natural Attenuation Field Experiment for an Ethanol-Blended Gasoline Spill, In: Proceedings of the 2000 Petroleum Hydrocarbons and Organic Chemicals in Ground Water: Prevention, Detection, and Remediation, Anaheim, California, 24-31.

Guy, E.D., Daniels, J.J., and Radzevicius, S.J., Demonstration of using crossed dipole GPR antennas for site characterization, Geophys. Res. Lett, 26, pp. 34213424 (1999).

Travassos, J. M., Simões, J. C., High-resolution radar mapping of internal layers of a subpolar ice cap, King George Island, Antarctica, Pesquisa Antártica Brasileira (Brazilian Antarctic Research), publ. Brazilian Academy of Sciences, vol. 4, 57-65 (2004).

Van Gestel, J-P., and Stoffa, P.L., Application of Alford Rotation to ground-penetrating radar data, Geophys., 66, 1781-1792 (2001). 